\title{
North American
}

National Cancer Institute

\section{Source}

National Cancer Institute. North American. NCI Thesaurus. Code C77812.

Denotes a person of European, Middle Eastern, or North African ancestral origins whose family settled in a North American country such as the United States of America or Canada. 